\title{
Highly Luminescent MgZnO/ZnO Multiple Quantum Wells for Photonics Devices
}

\author{
M. Zakria ${ }^{1}$, T. T. Huynh ${ }^{1}$, F. C. C. Ling $^{2}$, S. C. Su${ }^{2, \ddagger}$, M. R. Phillips ${ }^{1}$, C. Ton-That ${ }^{1, *}$ \\ ${ }^{1}$ School of Mathematical and Physical Sciences, University of Technology Sydney, Ultimo, \\ New South Wales 2007, Australia \\ ${ }^{2}$ Department of Physics, The University of Hong Kong, Pokfulam Road, Hong Kong, P. R. \\ China \\ * Corresponding author: cuong.ton-that@uts.edu.au \\ ‡ Current address: Institute of Optoelectronic Material and Technology, South China Normal \\ University, Guangzhou 510631, P. R. China
}

\begin{abstract}
Multiple quantum wells (MQWs) have enabled a myriad of technological applications; however, their optical emission is currently severely constrained by the presence of undesirable defects, which limit their performance in advanced photonic devices. Here we present a new route to achieve highly luminescent oxide-based MQWs by rapid remote plasma annealing (RRPA) in hydrogen. We demonstrate that the optical emission from the MgZnO/ZnO MQWs can be enhanced substantially by this plasma method, with its emission intensity increased by more than 10 times after being treated for 40 seconds. Concurrently, the emissions associated with both basal stacking faults and point defects are completely quenched. Based on temperature- and excitation density-dependent luminescence results, the enhancement of the MQW emission is attributed to the passivation of competitive recombination channels. Additionally, the exciton-optical phonon coupling strength, deduced from the temperaturedependent MQW spectral linewidth, shows clear evidence for significantly enhanced phonon coupling as a result of exciton screening effects. This rapid plasma procedure presents a versatile method to enhance the optical quality of oxide-based MQW structures and could open the door to high-efficiency photonic devices.
\end{abstract}


Keywords: multiple quantum wells; remote plasma; defects; cathodoluminescence; $\mathrm{ZnO;}$ photonics devices

\section{Introduction}

There has been considerable recent interest in developing optical devices based on zinc oxide $(\mathrm{ZnO})$, such as light emitting diodes, optically pumped ultraviolet lasers, nanoelectronic and spintronic devices, because of its attractive properties spanning from high exciton binding energy to radiation hardness. ${ }^{1-3}$ Among the variety of $\mathrm{ZnO}$-based materials, multiple quantum wells (MQWs) are of great interest because their emission wavelength can be fine-tuned for specific purposes by adjusting structural parameters. ${ }^{4} \mathrm{MQW}$, facilitated by the development of advanced growth techniques that allow for the precise control of 2D layer's stoichiometry and thickness, enable a myriad of existing and emerging technological applications. However, the optical emission efficiency of nitride- and oxide-based MQWs is currently severely constrained by the presence of undesirable point and extended defects that act as competitive recombination channels, which lead to non-radiative areas, loss in light output and shortened device lifetimes. ${ }^{5-7}$ To eradicate these unwanted recombination mechanisms and enhance the MQW optical emission, different approaches have been applied to the oxide-based MQWs, such as high-temperature $\left(>500{ }^{\circ} \mathrm{C}\right)$ thermal annealing or hydrogenation..$^{8-9}$ Due to its high chemical reactivity, hydrogen can interact with and passivate defect centres and dangling bonds at extended defects, as well as forms shallow donors in $\mathrm{ZnO} .{ }^{9-10}$ Hydrogen plasma treatment has been utilised to enhance the optical emission in InGaAs/AlGaAs, InGaN/GaN and CdZnO/ZnO MQWs. ${ }^{9,}{ }^{11-12}$ Here, we demonstrate a new route to achieve highly luminescent MQWs by rapid remote plasma annealing (RRPA), with the emission from the $\mathrm{MgZnO} / \mathrm{ZnO}$ MQWs being enhanced by more than 10 times. Importantly, our post-growth treatment 
procedure could open the door for new opportunities and directions for high-efficiency oxidebased photonics devices.

Previous optical spectroscopy studies have shown that the near-band-edge (NBE) emission in bulk and nanostructured $\mathrm{ZnO}$ can be significantly enhanced via atomic $\mathrm{H}$ incorporation. ${ }^{13-15}$ This increase in the NBE emission intensity has been attributed to (i) an additional radiative NBE channel at low temperatures due to the formation of H-donor-bound excitons, which are readily created due to the low formation energy of $\mathrm{H}$ donors in $\mathrm{ZnO}$ and (ii) due to the high diffusivity of $\mathrm{H}$ in $\mathrm{ZnO}$, and (iii) the passivation of competitive non-radiative recombination pathways in addition to radiative centres responsible for deep-level emissions. ${ }^{10}$, ${ }^{14,16}$ For example, $\mathrm{H}$ has been reported to form a stable complex consisting zinc vacancy $\left(V_{\mathrm{Zn}}\right)$ and two $\mathrm{H}$ atoms,${ }^{17}$ leading to the quenching of green luminescence at $2.3 \mathrm{eV} .{ }^{10}$ In this work, a new method for producing stable and highly luminescent $\mathrm{MgZnO} / \mathrm{ZnO}$ MQWs via lowtemperature hydrogen RRPA is investigated. A significant advantage of the RRPA method over traditional longer high-temperature thermal annealing is that $\mathrm{H}$ acts as an ultrafast diffusing species in $\mathrm{ZnO}$ even at low temperatures (diffusion speed $>10 \mathrm{~nm} / \mathrm{s}$ at $200{ }^{\circ} \mathrm{C}$ ), ${ }^{16,18}$ thus this method could be utilised to eliminate defects and improve the quality of optically active layers in MQWs without compromising the MQW structural integrity. We report here results from comprehensive characterization and theoretical modelling of RRPA-treated $\mathrm{MgZnO} / \mathrm{ZnO} \mathrm{MQWs}$ by employing temperature-, power density- and depth-dependent cathodoluminescence (CL) microanalysis.

\section{Experimental details}

The $\mathrm{MgZnO} / \mathrm{ZnO} \mathrm{MQWs}$ were grown on $a$-plane sapphire substrates by molecular beam epitaxy (MBE) at $500{ }^{\circ} \mathrm{C}$, starting with $75 \mathrm{~nm}$ thick $\mathrm{ZnO}$ buffer layer and finishing with $25 \mathrm{~nm} \mathrm{MgZnO}$ capping layer. The structure consists of 12 periods of $\mathrm{ZnO}(3 \mathrm{~nm}) / \mathrm{MgZnO}$ 
(6 nm) MQWs. The Mg composition in the barriers was 15 at. \%. RRPA was carried out via a post-growth annealing treatment at $200{ }^{\circ} \mathrm{C}$ in atomic $\mathrm{H}$ environment using a remote hydrogen plasma source (150 $\mathrm{W}$ power, $0.1 \mathrm{mbar}$ hydrogen pressure). After the RRPA, the sample was left to cool down to room temperature in $\mathrm{H}_{2}$ gas. High resolution transmission electron microscopy (HRTEM) was performed to investigate the MQWs interfaces using a JEOL 2010F TEM at $200 \mathrm{kV}$. CL was performed using a FEI Quanta scanning electron microscope (SEM) equipped with a custom-built CL spectroscopy and imaging system, which consists of a parabolic mirror, an Oriel MS257 monochromator and a Hamamatsu S7021 CCD sensor. This configuration provides a sub $0.1 \mathrm{~nm}$ spectral resolution. To probe the emission from different quantum wells, CL spectra were acquired over a range of acceleration voltages from 2 to $7 \mathrm{kV}$, corresponding to an excitation depth of $60-350 \mathrm{~nm}$ in $\mathrm{ZnO}$. The electron beam current was measured using a Faraday cup. All CL spectra of the pristine and RRPA-treated MQWs were acquired under identical conditions and corrected for the wavelength-dependent detection sensitivity of the system.

\section{Results and discussion}

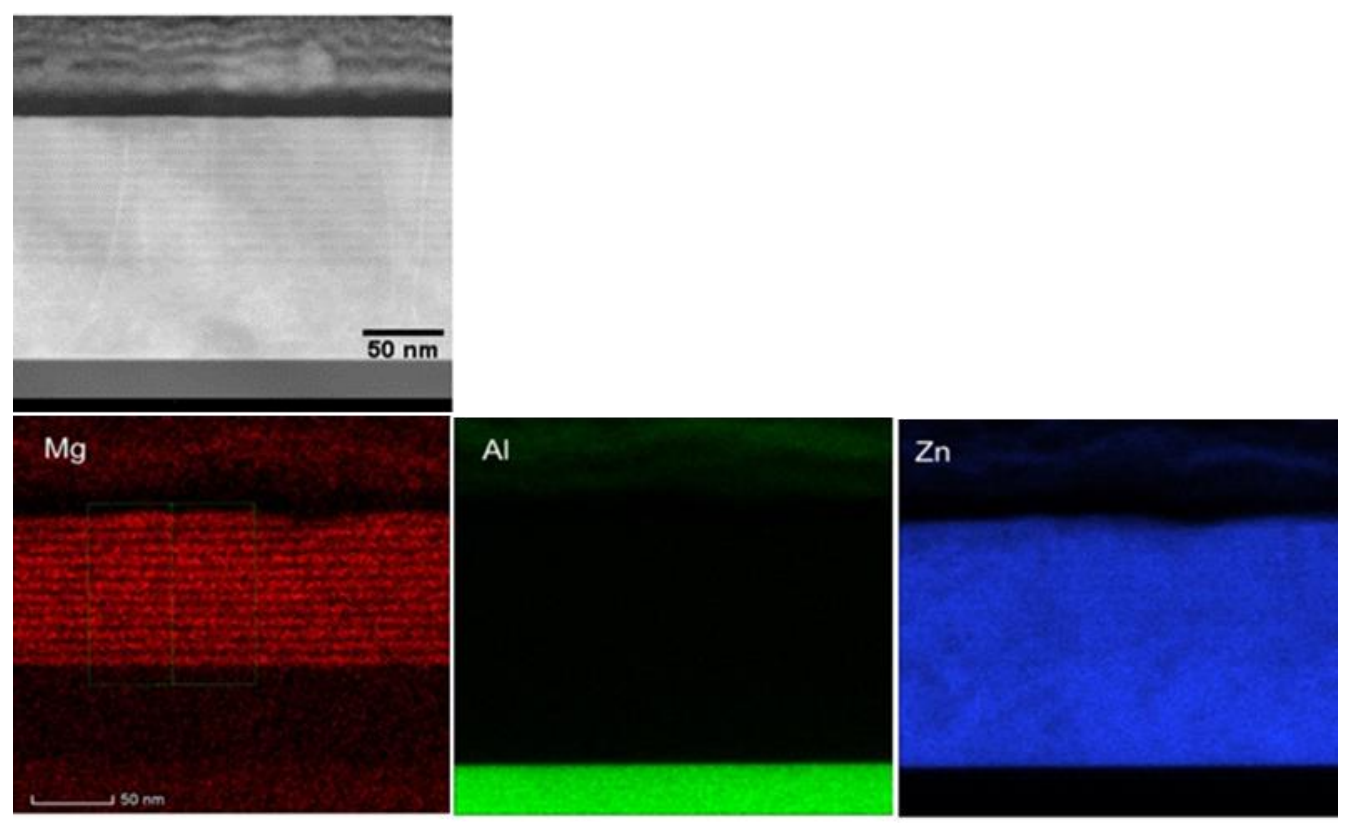


Fig. 1: Cross-sectional HRTEM image of the pristine MQW structure together with corresponding elemental energy-dispersive X-rays (EDX) maps of the $\mathrm{Mg}, \mathrm{Al}$ and $\mathrm{Zn}$ spatial distribution. The structure remains unchanged after the RRPA treatment.

Figure 1 shows a cross-sectional TEM image of the MQW structure with corresponding elemental EDX maps of the $\mathrm{Mg}, \mathrm{Al}$ and $\mathrm{Zn}$ spatial distribution. These results show that the interfaces between the well and barrier are flat and abrupt. The TEM analysis also confirms that the remote $\mathrm{H}$ plasma annealing has no effect on the MQW interfaces because of the low temperature employed in the RRPA. Figure 2(a) shows typical CL spectra measured at $80 \mathrm{~K}$ for the MQW structure, which was annealed in $\mathrm{H}$ remote plasma at $200{ }^{\circ} \mathrm{C}$ (denoted $\mathrm{H}: \mathrm{MQWs}$ ) and in Ar gas at the same temperature for 30 mins (denoted pristine MQWs). As $\mathrm{H}$ is known to exhibit rapid diffusion in $\mathrm{ZnO}$ at $200{ }^{\circ} \mathrm{C}, \mathrm{H}$ can easily migrate through the entire MQW structure to the $\mathrm{ZnO}$ buffer layer. ${ }^{16}$ Several types of radiative transitions can be clearly distinguished in the CL spectra shown in Figure 2: band-edge emission from the ZnO MQWs at $3.423 \mathrm{eV}$, basal stack fault (BSF) peak at $3.389 \mathrm{eV}$ and NBE emission from the $\mathrm{ZnO}$ buffer layer at $3.367 \mathrm{eV} \cdot{ }^{19-21}$ The BSF luminescence band, located at $34 \mathrm{meV}$ below the MQW emission energy, bears a strong resemblance to the luminescence originated from embedded zinc-blende structure within the wurtzite MQWs. ${ }^{20,22}$ Excitation-power and depth-resolved CL analysis of the pristine and RRPA-treated MQWs further confirms that the MQW and NBE emissions are of excitonic nature and not related to a lattice coupled defect (See Figs S1 and S2 in Supporting Information). Upon the RRPA, the MQW emission increases rapidly with the $\mathrm{H}$ plasma exposure time, reaching an 11-fold intensity enhancement after a $40 \mathrm{sec}$ treatment. At $V_{\mathrm{B}}=2 \mathrm{kV}, e-h$ pairs are injected with a maximum depth range of $60 \mathrm{~nm}$ in the MQW structure, which is well above the $\mathrm{ZnO}$ buffer layer, thus none of the injected carriers can physically reach the buffer layer to radiatively recombine. Accordingly, the ZnO NBE emission is attributed to the buffer layer being excited by the forward generated MQW CL emission; this 
reabsorption process within the MQW structure leads to these two bands to vary in proportion to each other. Conversely, the BSF emission, as well as the defect-related green luminescence band at $2.3 \mathrm{eV}$ (inset of Fig. 2b), are completely quenched. Our previous studies have shown that the elimination of this defect-related luminescence is consistent with the passivation of acceptor-like defects, mostly likely $V_{\mathrm{Zn}}$, by $\mathrm{H}$ atoms ${ }^{10}$ and confirms that $\mathrm{H}$ is incorporated into all the QWs and the buffer layer after the RRPA treatment. Previous studies on BSF-related luminescence have shown that the BSF emission could be ascribed as an acceptor-like free-tobound transition, though the exact nature of the acceptor is unknown. ${ }^{23}$ Such an acceptor would interact strongly with $\mathrm{H}^{+}$donors, leading to the BSF emission being completely eliminated by the RRPA. It was found that that the low-temperature RRPA method is far superior in enhancing the MQW emission to high-temperature annealing in hydrogen, presumably due to the high reactivity of $\mathrm{ZnO}$ to hydrogen radicals produced by the remote plasma [Figures S3(a)]. Furthermore, the luminescence emission in the RRPA-treated MQWs is completely stable in ambient conditions for over 6 months and reduces only slightly after annealing at $400{ }^{\circ} \mathrm{C}$ for 1 hour [Figure S3(b)]. The high thermal stability of the RRPA-treated MQWs, compared with $\mathrm{H}$-doped $\mathrm{ZnO}$ bulk, ${ }^{24}$ suggests that hydrogen radicals incorporated into the MQW structure by the RRPA are mainly trapped at defect sites, which possess a much higher diffusion activation energy than interstitial sites. ${ }^{25-26}$ Fig. $2 \mathrm{~b}$ shows the integrated MQW emission versus H plasma time, revealing a rapid rise in intensity followed by a relatively slower decline after reaching a maximum at a plasma exposure time of 40 seconds. The reduction in the MQW emission with longer H-plasma exposure time is attributed to a surface degradation effect (see the AFM images of the MQW structure before and after RRPA in Fig. S4). The formation of near-surface structural defects in prolonged plasma treatments lowers the MQW intensity as they act as competitive recombination channels. 

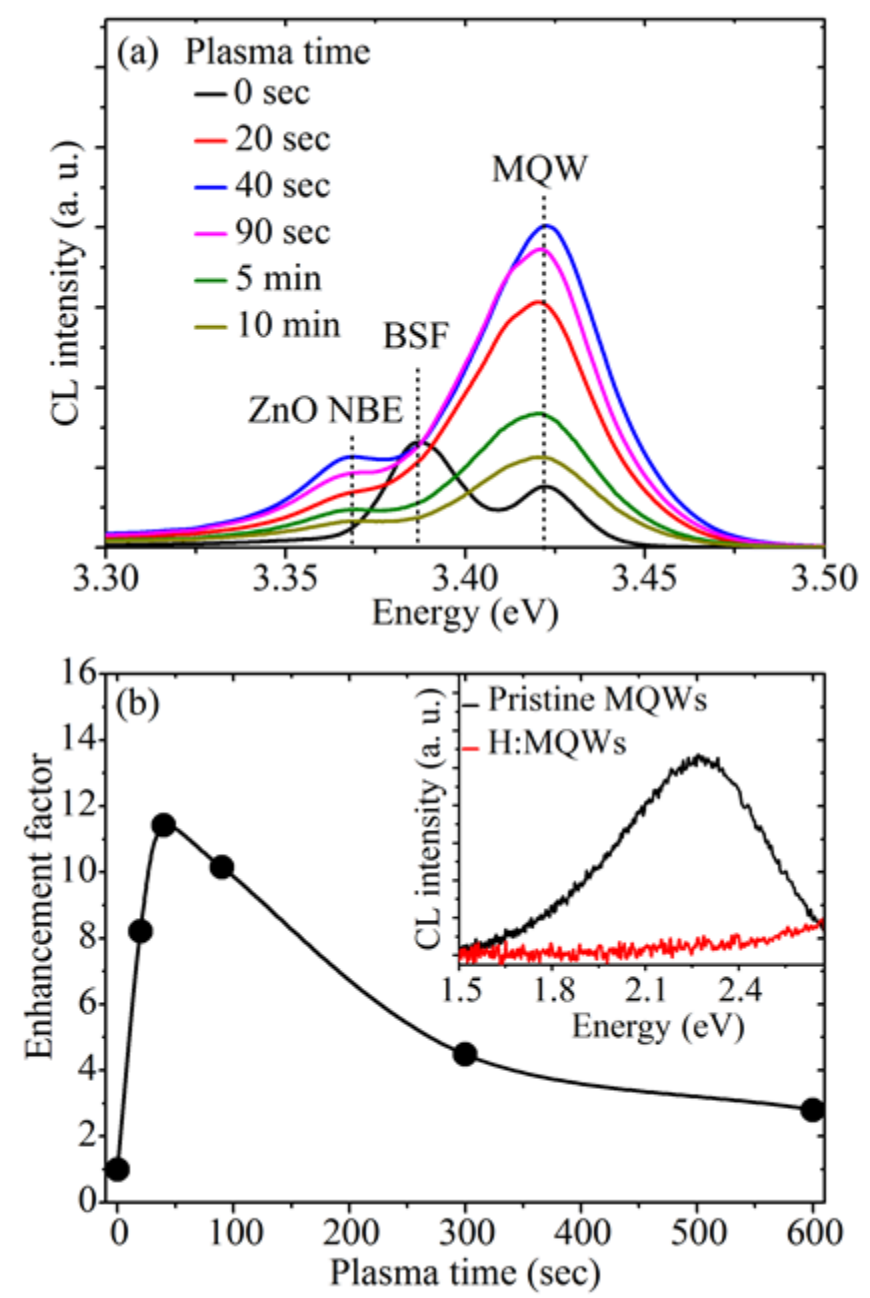

Fig. 2: (a) CL spectra of pristine and RRPA-treated $\mathrm{MgZnO} / \mathrm{ZnO} \mathrm{MQWs}\left(V_{\mathrm{B}}=2 \mathrm{kV}, I_{\mathrm{B}}=3.8\right.$ $\mathrm{nA}, T=80 \mathrm{~K}$ ), showing a substantial increase in the MQW emission and complete elimination of the BSF emission upon the $\mathrm{H}$ introduction. The emergence of the $\mathrm{NBE} \mathrm{ZnO}$ emission at $3.367 \mathrm{eV}$ is due to the formation of $\mathrm{H}$ donor-bound excitons in bulk $\mathrm{ZnO}$. (b) Enhancement factor (defined as the MQW intensity ratio of the RRPA-treated MQW structure to the pristine structure) as a function of plasma time, showing a rapid rise in intensity to reach an 11-fold enhancement after $40 \mathrm{sec}$ plasma treatment. The decrease in the MQW emission after prolonged plasma treatments is due to surface damage. Inset: CL spectra of the defect-related green emission at $2.3 \mathrm{eV}$ from the pristine MQWs, which is completely quenched after the RRPA treatment for 40 seconds or longer. 

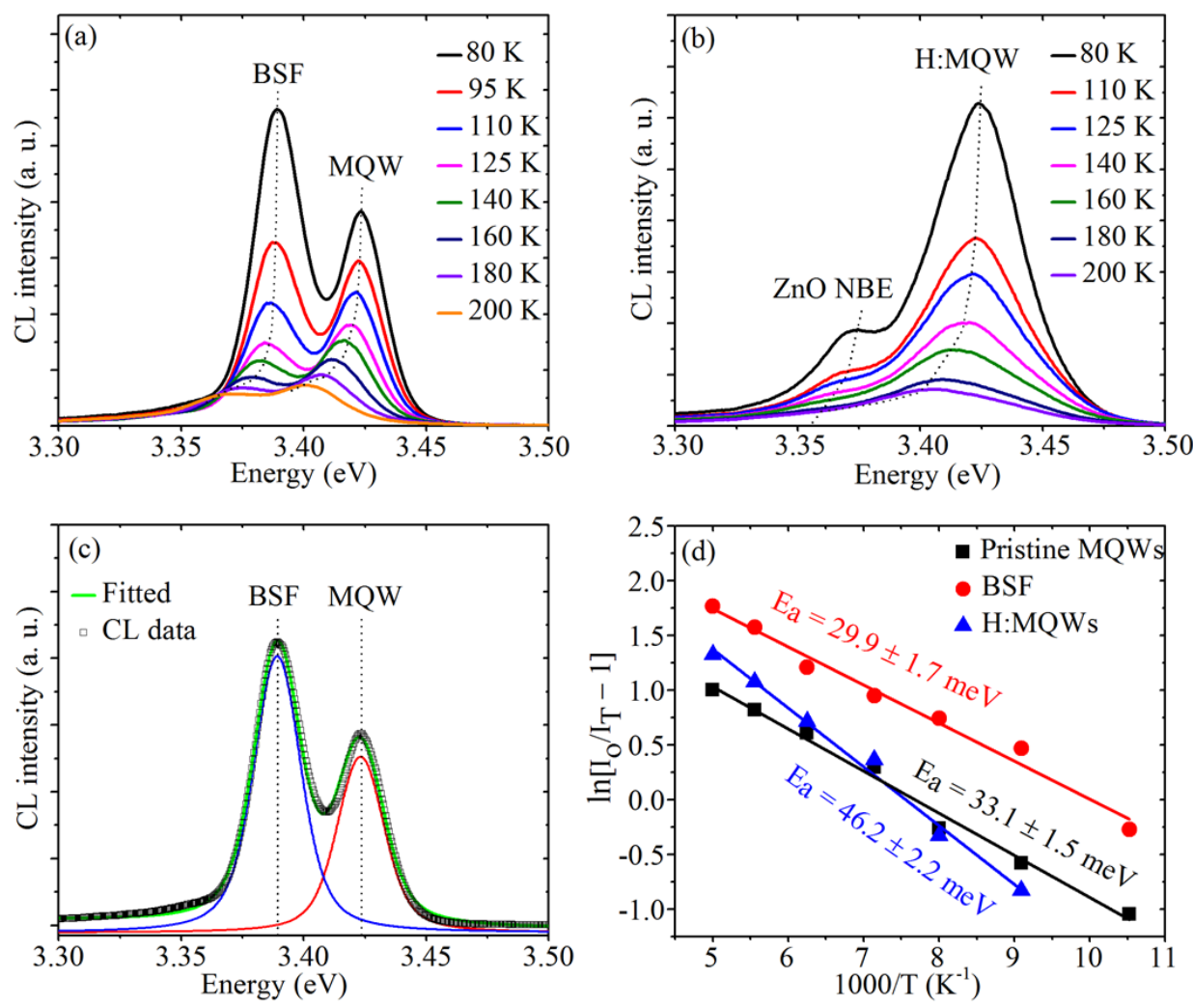

Fig. 3: Temperature-resolved CL spectra of the pristine (a) and $40 \mathrm{sec}$ plasma treated MQW structures (b) $\left(V_{\mathrm{B}}=2 \mathrm{kV}, I_{\mathrm{B}}=3.8 \mathrm{nA}\right)$ at various temperatures from $80 \mathrm{~K}$. (c) Example of a fitted CL spectrum, allowing extraction of the BSF and MQW integrated intensities and FWHMs as a function of temperature. (d) Arrhenius plots using integrated intensities, yielding $E_{\mathrm{a}}=29.9 \pm 1.7 \mathrm{meV}$ for the BSF emission, $E_{\mathrm{a}}=33.1 \pm 1.5 \mathrm{meV}$ and $46.2 \pm 2.2 \mathrm{meV}$ for the pristine and RRPA-treated MQWs, respectively.

The evolution of the CL spectra with temperature for the pristine and RRPA-treated MQWs is displayed in Figure 3(a,b). Voigt fitting of the CL spectra provides the integrated intensities of the ZnO NBE, BSF and MQW emission bands; an example of the fitting deconvolution is displayed in Figure 3(c). Thermal quenching of these luminescence bands with increasing temperature follows an Arrhenius behaviour with activation energies of $E_{\mathrm{a}}=$ 33.1 \pm 1.5. $46.2 \pm 2.2 \mathrm{meV}$ for the MQW emission in the pristine and RRPA-treated MQW 
structure, respectively [see Figure 3(d)]. The $E_{\mathrm{a}}(29.9 \pm 1.7 \mathrm{meV})$ of the BSF emission is consistent with the reported value for non-polar $\mathrm{MgZnO} / \mathrm{ZnO} \mathrm{MQWs} .{ }^{20}$ Furthermore, this $E_{\mathrm{a}}$ value is notably similar to that of the MQW band in the pristine MQWs, suggesting that the thermal activation of carriers is controlled by the thermal activation of competitive nonradiative centres, which is expected for MQW structures prior to the $\mathrm{H}$ passivation of defects. The activation energy of the RRPA-treated MQW emission is identical to the binding energy of $\mathrm{H}$ donors in $\mathrm{ZnO},{ }^{27}$ pointing towards the activation of a dominant recombination channel related to the ionization of $\mathrm{H}$ donors. It can be seen in Fig. 3(a, b) that the MQW emission peak becomes broader after the RRPA, which can be attributed to an increase in the exciton-phonon interaction strength. Figure 4 shows the temperature dependence of the FWHM of the MQW emission for the pristine and RRPA-treated MQW structures. Based on Segall's expression for the temperature-dependent linewidth of excitonic emission, the FWHM can be described by: ${ }^{28}$

$$
\beta(T)=\beta_{i n h}+\beta_{p h} T+\frac{\beta_{L O}}{\exp \left(\hbar \omega_{L O} / k_{B} T\right)-1}
$$

where $\beta_{\text {inh }}$ is the temperature-independent term describing the inhomogeneous linewidth accounting for the scattering of impurities, defects and well width fluctuations. The second term is associated with the acoustic phonon scattering, which is vanishingly small compared with the LO scattering and can be safely ignored at $T>80 \mathrm{~K} .{ }^{29}$ The third term is the linewidth due to the LO phonon scattering, which dominates the phonon scattering at $T>80 \mathrm{~K}\left(\beta_{L O}\right.$ and $\hbar \omega_{L O}$ are the phonon coupling strength and LO phonon energy, respectively). As the energy $\hbar \omega_{L O}$ has been shown to be unchanged with the MQW well width, ${ }^{30}$ we used $\hbar \omega_{L O}=72 \mathrm{meV}$ in the curve fitting procedure for temperature-dependent MQW linewidths. The curves in Figure 4 represent the best fit to the FWHM data based on Eq. [1], yielding $\beta_{\text {inh }}=17 \mathrm{meV}$ and $\beta_{L O}=130 \mathrm{meV}$ for the pristine MQWs, and $\beta_{\text {inh }}=25 \mathrm{meV}$ and $\beta_{L O}=371 \mathrm{meV}$ for the 40 second RRPA-treated MQWs. As expected, the $\beta_{\text {inh }}$ value becomes larger after the RRPA 
due to additional scattering by $\mathrm{H}$ impurities and $\mathrm{H}$-defect complexes. The notable increase in $\beta_{L O}$ in the MQWs after the RRPA indicates a much stronger exciton-LO phonon coupling process in the optical emission of the RRPA-treated MQW structure. For comparison, $\beta_{L O}$ is $250 \mathrm{meV}$ for MBE-grown undoped MQWs with a similar well width on the lattice-matched substrate. ${ }^{7}$ Previous studies by other workers have shown that as the QW well thickness becomes larger, $\beta_{L O}$ increases due to lowered exciton binding energy. ${ }^{29}$ The exceptionally high $\beta_{L O}$ in the RRPA-treated MQWs can be attributed to a reduction in the exciton binding energy due to the enhanced screening of excitons by free electrons that are produced by $\mathrm{H}$ donors in $\mathrm{ZnO}$.

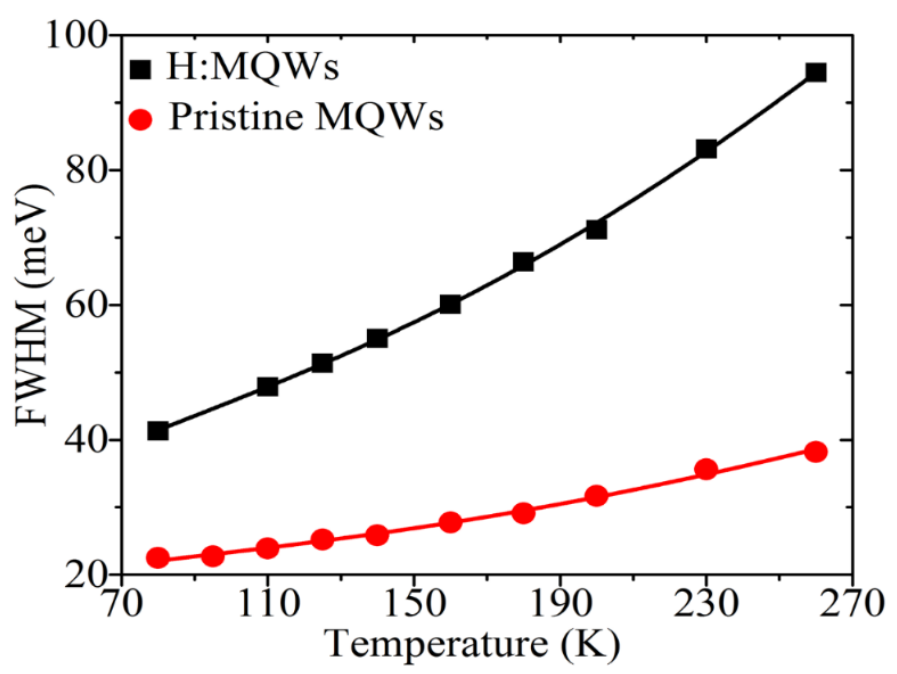

Fig. 4: FWHM of the MQW emission from the pristine and RRPA-treated MQW structures. The solid line represent the fit to the experimental data according to Eq. [1]. The larger FWHM in the RRPA-treated MQW structure is due to enhanced exciton-phonon coupling strength.

To probe the spatial distribution of emission in the QWs, depth-resolved CL was conducted in the pristine and RRPA-treated MQW structures. In this measurement the electron beam power and hence the injected electron-hole (e-h) pair rate in the sample was kept constant by adjusting the electron beam current as the accelerating voltage was raised to increase the 
CL excitation depth. Monte Carlo modelling was carried out using the CASINO simulation package to determine the spatial distribution of the injected electron hole pairs in the MQWs for the acceleration voltages between 2 and $7 \mathrm{kV}$ (Figure 5a). ${ }^{31}$ The depth profiles reveal that at $V_{\mathrm{B}}=2 \mathrm{kV}$ the measured MQW emission is generated only from the top four QWs, with the upper QWs contributing a stronger MQW signal compared with the bottom QWs. At $V_{\mathrm{B}}=4-$ $5 \mathrm{kV}$ the MQW optical emission is contributed fairly evenly by all $12 \mathrm{QWs}$, while $V_{\mathrm{B}}>6 \mathrm{kV}$ an increasing number of e-h pairs are generated within the $\mathrm{ZnO}$ buffer layer. Figure 5(b) shows the measured luminescence enhancement factor, which is defined as the ratio of the MQW emission intensities from the RRPA-treated MQWs to the pristine MQWs, as a function of $V_{\mathrm{B}}$. A second $x$-axis (top) is also shown to display the CL excitation depth. The enhancement factor increases with $V_{\mathrm{B}}$ up to $4 \mathrm{kV}$, which is then followed by a slow decline after reaching a maximum value of 17-fold enhancement. The highest MQW enhancement factor achieved at $V_{\mathrm{B}}=4-5 \mathrm{kV}$ confirms that $\mathrm{H}$ diffuses into all the QWs. The surface degradation caused by hydrogen plasma could contribute to low enhancement factors in the top four QWs. Another consideration is that $\mathrm{H}$ could diffuse out from top QWs, which cannot be ruled out. The decline in the enhancement factor at $V_{\mathrm{B}}>5 \mathrm{kV}$ could be due to self-absorption effects as photons from deep QWs are absorbed by upper QWs. 

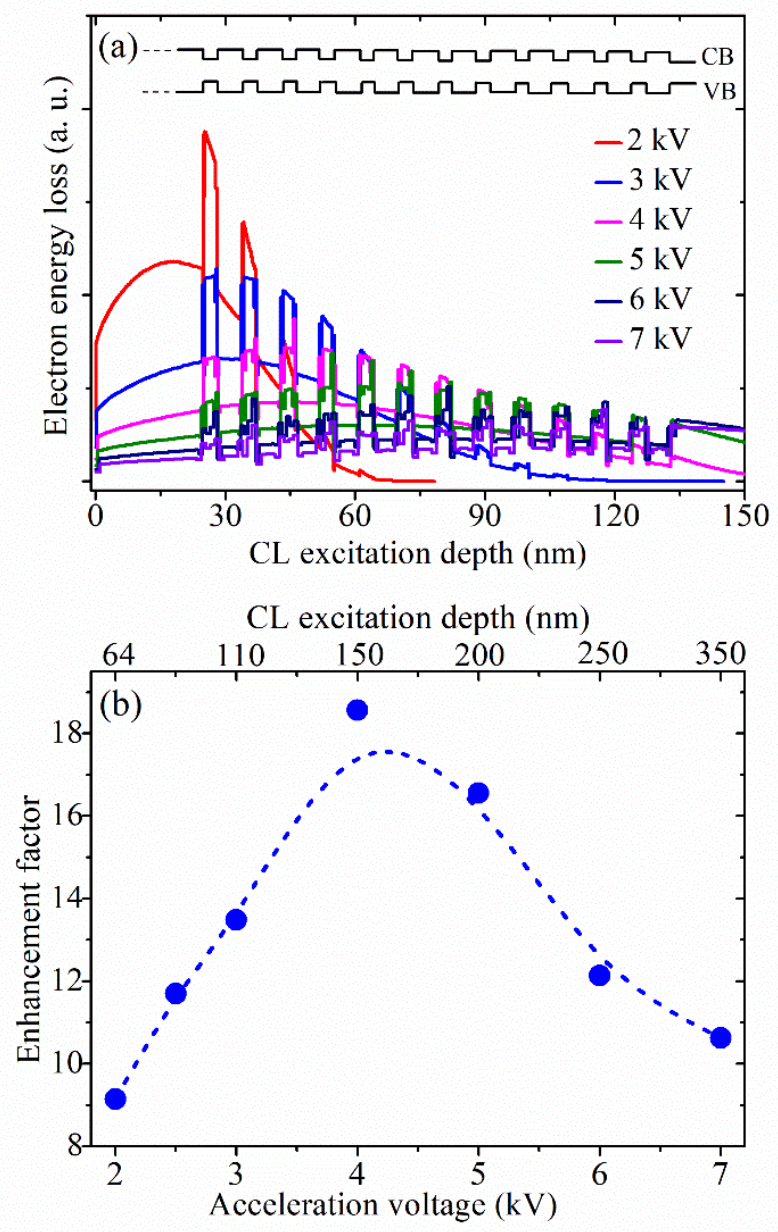

Fig. 5: (a) Simulated electron energy loss curves generated in the MQWs for $V_{\mathrm{B}}=2-7 \mathrm{kV}$, showing the e-beam reaches all $12 \mathrm{QWs}$ at $4 \mathrm{kV}$. Inset shows the schematic diagram of the valence and conduction bands for the MQWs. (b) Enhancement factor as functions of acceleration voltage and CL excitation depth. The maximum enhancement is achieved at $V_{\mathrm{B}}=$ $4-5 \mathrm{kV}$ when the optical emission is contributed fairly evenly by all $12 \mathrm{QWs}$.

\section{Conclusion}

We have studied effects of the incorporation of $\mathrm{H}$ via rapid remote plasma annealing treatment on the optical properties and defect emissions in $\mathrm{MgZnO} / \mathrm{ZnO} \mathrm{MQWs}$. Comprehensive cathodoluminescence analysis reveals the plasma method can lead to a substantial improvement (over 10 times) in the emission intensity of the MQWs after being treated for 40 seconds. Based on the CL kinetics and temperature-dependent measurements, 
the enhancement is attributed to the passivation of competitive recombination channels, in particular point defects and stacking faults in the MQW structure. Theoretical modelling of the MQW spectral linewidth indicates that the exciton and longitudinal optical phonon coupling process is much stronger in the plasma-treated MQWs, which is attributed to exciton screening effects. This rapid plasma annealing presents a facile, effective method to enhance the optical quality of oxide-based MQW structures.

\section{ASSOCIATED CONTENT}

\section{Supporting Information}

The Supporting Information is available free of charge on the ACS Publications website at DOI:

Additional results from CL analysis of the RRPA-treated MQWs and AFM images of the MQW structure (PDF)

\section{AUTHOR INFORMATION}

\section{Corresponding Author}

*E-mail: cuong.ton-that@uts.edu.au

\section{ORCID}

Cuong Ton-That: 0000-0003-3276-1739

\section{Author Contributions}

The manuscript was written through contributions of all authors. All authors have given approval to the final version of the manuscript.

\section{Notes}

The authors declare no competing financial interest. 


\section{Acknowledgments}

This work was supported under Australian Research Council (ARC) Discovery Project funding scheme (project number DP150103317). We would like to thank Olivier Lee for assistance with the CL measurements.

\section{References}

1. Kang, J. W.; Kim, B. H.; Song, H.; Jo, Y. R.; Hong, S. H.; Jung, G. Y.; Kim, B. J.; Park, S. J.; Cho, C. H., Radial multi-quantum well $\mathrm{ZnO}$ nanorod arrays for nanoscale ultraviolet light-emitting diodes. Nanoscale 2018, 10, 14812-14818.

2. Sidiropoulos, T. P. H.; Roder, R.; Geburt, S.; Hess, O.; Maier, S. A.; Ronning, C.; Oulton, R. F., Ultrafast plasmonic nanowire lasers near the surface plasmon frequency. Nat. Phys. 2014, 10, 870-876.

3. Sun, Z. H.; Yang, X. Y.; Wang, C.; Yao, T.; Cai, L.; Yan, W. S.; Jiang, Y.; Hu, F. C.; He, J. F.; Pan, Z. Y.; Liu, Q. H.; Wei, S. Q., Graphene Activating Room-Temperature Ferromagnetic Exchange in Cobalt-Doped ZnO Dilute Magnetic Semiconductor Quantum Dots. ACS Nano 2014, 8, 10589-10596.

4. Morhain, C.; Bretagnon, T.; Lefebvre, P.; Tang, X.; Valvin, P.; Guillet, T.; Gil, B.; Taliercio, T.; Teisseire-Doninelli, M.; Vinter, B.; Deparis, C., Internal electric field in wurtzite $\mathrm{ZnO} / \mathrm{Zn0.78Mg0.22O} \mathrm{quantum} \mathrm{wells.} \mathrm{Phys.} \mathrm{Rev.} \mathrm{B} \mathrm{2005,} \mathrm{72,} 4$.

5. Chang, M. H.; Das, D.; Varde, P. V.; Pecht, M., Light emitting diodes reliability review. Microelectron. Reliab. 2012, 52, 762-782.

6. Chauveau, J.-M.; Teisseire, M.; Kim-Chauveau, H.; Deparis, C.; Morhain, C.; Vinter, B., Benefits of homoepitaxy on the properties of nonpolar $(\mathrm{Zn}, \mathrm{Mg}) \mathrm{O} / \mathrm{ZnO}$ quantum wells on a-plane ZnO substrates. Appl. Phys. Lett. 2010, 97, 081903. 
7. Davis, J. A.; Jagadish, C., Ultrafast spectroscopy of $\mathrm{ZnO} / \mathrm{ZnMgO}$ quantum wells. Laser Photon. Rev. 2009, 3, 85-96.

8. Thompson, A. V.; Boutwell, C.; Mares, J. W.; Schoenfeld, W. V.; Osinsky, A.; Hertog, B.; Xie, J. Q.; Pearton, S. J.; Norton, D. P., Thermal stability of CdZnO/ZnO multiquantum-wells. Appl. Phys. Lett. 2007, 91, 3.

9. $\quad$ Buyanova, I.; Wang, X.; Pozina, G.; Chen, W.; Lim, W.; Norton, D.; Pearton, S.; Osinsky, A.; Dong, J.; Hertog, B., Effects of hydrogen on the optical properties of Zn Cd O/ Zn O quantum wells grown by molecular beam epitaxy. Appl. Phys. Lett. 2008, 92, 261912. 10. Ton-That, C.; Weston, L.; Phillips, M., Characteristics of Point Defects in the Green Luminescence from Zn-and O-rich ZnO. Phys. Rev. B 2012, 86, 115205.

11. Ren, P.; Zhang, N.; Xue, B.; Liu, Z.; Wang, J.; Li, J., A novel usage of hydrogen treatment to improve the indium incorporation and internal quantum efficiency of green InGaN/GaN multiple quantum wells simultaneously. J. Phys. D: Appl. Phys. 2016, 49, 175101.

12. Lord, S.; Roos, G.; Harris Jr, J.; Johnson, N., Hydrogen passivation of nonradiative defects in InGaAs/Al x Ga1- x As quantum wells. J. Appl. Phys. 1993, 73, 740-748.

13. Lin, C. C.; Chen, H. P.; Liao, H. C.; Chen, S. Y., Enhanced luminescent and electrical properties of hydrogen-plasma $\mathrm{ZnO}$ nanorods grown on wafer-scale flexible substrates. Appl. Phys. Lett. 2005, 86, 183103.

14. Dev, A.; Niepelt, R.; Richters, J. P.; Ronning, C.; Voss, T., Stable enhancement of near-band-edge emission of $\mathrm{ZnO}$ nanowires by hydrogen incorporation. Nanotechnology 2010, 21, 065709.

15. Lem, L. C. L.; Ton-That, C.; Phillips, M. R., Distribution of visible luminescence centers in hydrogen-doped ZnO. J. Mater. Res. 2011, 26, 2912-2915. 
16. Ip, K.; Overberg, M. E.; Heo, Y. W.; Norton, D. P.; Pearton, S. J.; Stutz, C. E.; Kucheyev, S. O.; Jagadish, C.; Williams, J. S.; Luo, B.; Ren, F.; Look, D. C.; Zavada, J. M., Hydrogen incorporation, diffusivity and evolution in bulk ZnO. Solid-State Electron. 2003, 47, 2255-2259.

17. Lavrov, E. V.; Weber, J.; Borrnert, F.; Van de Walle, C. G.; Helbig, R., Hydrogenrelated defects in $\mathrm{ZnO}$ studied by infrared absorption spectroscopy. Phys. Rev. B 2002, 66, 165205.

18. Rodrigues, J.; Holz, T.; Allah, R. F.; Gonzalez, D.; Ben, T.; Correia, M. R.; Monteiro, T.; Costa, F. M., Effect of N 2 and H 2 plasma treatments on band edge emission of ZnO microrods. Sci. Rep. 2015, 5, 10783.

19. Ashrafi, A., Exciton localization in inhomogeneously broadened $\mathrm{ZnO} / \mathrm{MgxZn1-xO}$ quantum wells. J. Appl. Phys. 2010, 107, 5.

20. Chen, H.; Tsai, C.; Huang, Y.; Kuo, C.; Hsu, H.; Hsieh, W., Optical Properties of One-and Two-dimensional Excitons in M-plane ZnO/MgZnO Multiple Quantum Wells. J. Phys. D: Appl. Phys. 2016, 49, 095105.

21. Makino, T.; Chia, C.; Tuan, N. T.; Sun, H.; Segawa, Y.; Kawasaki, M.; Ohtomo, A.; Tamura, K.; Koinuma, H., Room-temperature luminescence of excitons in $\mathrm{ZnO} /(\mathrm{Mg}, \mathrm{Zn}) \mathrm{O}$ multiple quantum wells on lattice-matched substrates. Appl. Phys. Lett. 2000, 77, 975-977. 22. Schirra, M.; Schneider, R.; Reiser, A.; Prinz, G.; Feneberg, M.; Biskupek, J.; Kaiser, U.; Krill, C.; Thonke, K.; Sauer, R., Stacking fault related 3.31-eV luminescence at 130-meV acceptors in zinc oxide. Phys. Rev. B 2008, 77, 125215.

23. Urgessa, Z. N.; Botha, J. R.; Eriksson, M. O.; Mbulanga, C. M.; Dobson, S. R.; Djiokap, S. R. T.; Karlsson, K. F.; Khranovskyy, V.; Yakimova, R.; Holtz, P. O., Low temperature near band edge recombination dynamics in $\mathrm{ZnO}$ nanorods. J. Appl. Phys. 2014, $116,10$. 
24. Ip, K.; Overberg, M. E.; Heo, Y. W.; Norton, D. P.; Pearton, S. J.; Kucheyev, S. O.; Jagadish, C.; Williams, J. S.; Wilson, R. G.; Zavada, J. M., Thermal Stability of Ionimplanted Hydrogen in ZnO. Appl. Phys. Lett. 2002, 81, 3996-3998.

25. Wardle, M. G.; Goss, J. P.; Briddon, P. R., First-principles Study of the Diffusion of Hydrogen in ZnO. Phys. Rev. Lett. 2006, 96, 205504.

26. Nickel, N. H., Hydrogen Migration in Single Crystal and Polycrystalline Zinc Oxide. Phys. Rev. B 2006, 73, 9.

27. Meyer, B. K.; Alves, H.; Hofmann, D. M.; Kriegseis, W.; Forster, D.; Bertram, F.; Christen, J.; Hoffmann, A.; Strassburg, M.; Dworzak, M.; Haboeck, U.; Rodina, A. V., Bound exciton and donor-acceptor pair recombinations in ZnO. Phys. Status Solidi B-Basic Res. 2004, 241, 231-260.

28. Rudin, S.; Reinecke, T. L.; Segall, B., Temperature-dependent Exciton Linewidths in Semiconductors. Phys. Rev. B 1990, 42, 11218-11231.

29. Makino, T.; Segawa, Y.; Kawasaki, M.; Koinuma, H., Optical properties of excitons in ZnO-based quantum well heterostructures. Semicond. Sci. Technol. 2005, 20, S78-S91.

30. Sun, H.; Makino, T.; Tuan, N.; Segawa, Y.; Kawasaki, M.; Ohtomo, A.; Tamura, K.; Koinuma, H., Temperature dependence of excitonic absorption spectra in $\mathrm{ZnO} / \mathrm{Zn} 0.88 \mathrm{Mg}$ $0.12 \mathrm{O}$ multiquantum wells grown on lattice-matched substrates. Appl. Phys. Lett. 2001, 78, 2464-2466.

31. Drouin, D.; Hovington, P.; Gauvin, R., CASINO: A new monte carlo code in C language for electron beam interactions - part II: Tabulated values of the mott cross section. Scanning 1997, 19, 20-28. 
Abstract Graphic
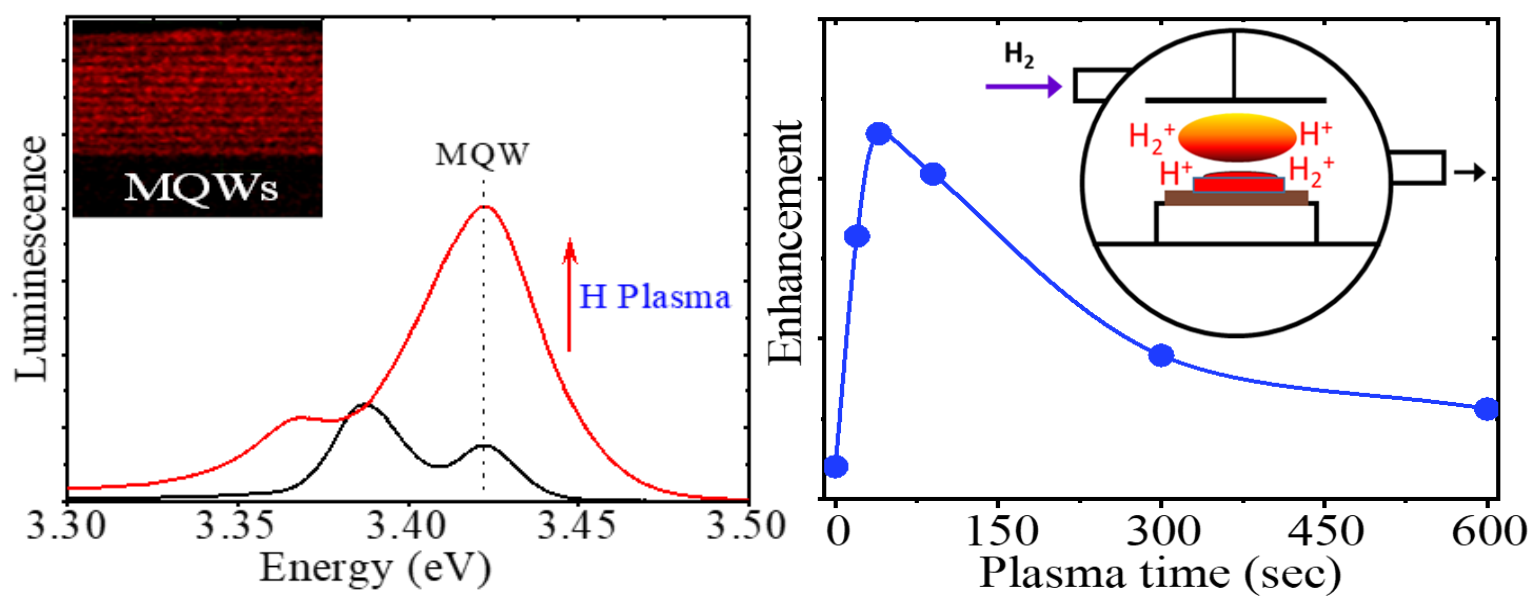